\title{
ESTUDIO DE LAS EMISIONES DE GASES DE EFECTO INVERNADERO PRODUCIDOS POR LOS EMBALSES DE LAS CENTRALES HIDROELÉCTRICAS DEL PERÚ
}

\author{
STUDY OF THE EMISSIONS OF GREENHOUSE GASES PRODUCED BY \\ THE PACKAGES OF THE HYDROELECTRIC POWER STATIONS OF \\ PERU
}

\author{
Reynaldo Villanueva Ure ${ }^{1^{*}(\mathbb{D})}$, Elmar Javier Franco Gonzales ${ }^{1}(\mathbb{D}$, \\ Gilberto Becerra Arévalo' ${ }^{1(i)}$
}

'Universidad Nacional de Ingeniería, Facultad de Ingeniería Mecánica, Lima, Perú

Recibido (Received): 04 / 03/2020 Aceptado (Accepted): 11/08 / 2020

\section{RESUMEN}

El proceso de generación hidroeléctrica se compone esencialmente de tres etapas que son la del embalse de agua, la de transformación de energía hidráulica a energía eléctrica y la de entrega de la energía eléctrica para su distribución. En la etapa del embalse se realizó la cuantificación de emisiones de gases de efecto invernadero usando la metodología propuesta por la Organización de las Naciones Unidas, denominada Panel Intergubernamental para el Cambio Climático del 2006 en diez centrales hidroeléctricas peruanas. Se concluyó que, en la primera fase de la generación hidroeléctrica, que es la del embalse, la contaminación significativa total de dióxido de carbono, CO2, llegó a ser 83947 toneladas por año y se contrastó los resultados comparando con datos obtenidos de embalses similares de hidroeléctricas brasileñas.

Palabras Clave: Emisiones, Embalses, Hidroeléctricas, Perú, Brasil

\section{ABSTRACT}

The hydroelectric generation process is essentially composed of three stages that are the water reservoir, the transformation of hydraulic energy to electrical energy and the delivery of electrical energy for distribution. In the reservoir stage, the quantification of greenhouse gas emissions was carried out using the methodology proposed by the United Nations Organization, called the 2006 Intergovernmental Panel for Climate Change in ten Peruvian hydroelectric plants. It was concluded that, in the first phase of hydroelectric generation, which is that of the reservoir, the total significant contamination of carbon dioxide, $\mathrm{CO}$, reached 83947 tons per year and the results were compared by comparing data obtained from Brazilian hydroelectric reservoirs.

Keywords: Emissions, Reservoirs, Hydroelectric, Peruvian, Brazilian

\section{INTRODUCCIÓN}

En los embalses de las centrales de generación de energía eléctrica, se produce el contacto entre el agua embalsada y la tierra generando una mezcla de barro, lodo y restos orgánicos llamo "limo" el cual actúa como fertilizante natural para el crecimiento de las algas y fitoplancton los cuales realizan oxidación aeróbica, lo cual genera un cambio en la calidad del agua cambiando sus características químicas, físicas,

\footnotetext{
${ }^{1^{*}}$ Corresponding author.:

E-mail: rvillauevaaure@yahoo.es
}

biológicas y radiológicas que constituye el origen de la contaminación atmosférica por emisión de $\mathrm{CO}_{2}$ y $\mathrm{CH}_{4}$ principalmente. [1]

Del total de las centrales hidroeléctricas del Perú se seleccionó diez de las más significativas, a manera de muestra, teniendo en cuenta la mayor producción de energía eléctrica, las cuales representan el 17.74 \% de producción de energía eléctrica en el país.

De cinco metodologías disponibles para calcular la cantidad de $\mathrm{CO}_{2}$ y $\mathrm{CH}_{4}$ se seleccionó la proporcionada por la Organización de las Naciones Unidas denominada la Metodología del Panel Intergubernamental para el cambio Climático (IPCC-2006), dada la confiabilidad de su eficacia en la cuantificación de gases de efecto 
invernadero mostrada en centrales hidroeléctricas, así como de las mediciones y estimaciones existentes en todos sus años de uso. [2]

Se midió el área inundada en hectáreas, de cada una de las diez centrales hidroeléctricas seleccionadas y se recopilo información relacionada al período de hielo, de la fracción del área total del reservorio que se inundó en los últimos diez años, del período diario de las emisiones de monóxido de carbono y metano.

Con dicha información se pudo obtener los valores de las emisiones totales de dióxido de carbono equivalente $\left(\mathrm{CO}_{2}\right)$ y de metano equivalente $\left(\mathrm{CH}_{4}\right)$ correspondientes a cada una de las centrales de generación hidroeléctrica.

Los resultados obtenidos en los cálculos de las emisiones de dióxido de carbono y metano de las diez centrales hidroeléctricas peruanas que fueron seleccionadas se compararon con los valores de emisiones de diez centrales hidroeléctrica similares del Brasil y con el valor estándar de emisiones de $\mathrm{CO}_{2}$ dadas por la Organización de las Naciones Unidas (ONU). Con respecto a las centrales hidroeléctricas brasileñas, el porcentaje de las emisiones de dióxido de carbono de las centrales hidroeléctricas peruanas es del $5 \%$ y con respecto al estándar de la ONU, es del 6.6\%, siendo 0.02 Ton $\mathrm{CO}_{2} / \mathrm{KW}$ la emisión de $\mathrm{CO}_{2}$ en el Perú.

\section{MARCO TEÓRICO}

\subsection{GASES DE EFECTO INVERNADERO}

El efecto invernadero es un fenómeno natural que se da en nuestro planeta debido a que al haber ciertos gases en la frontera entre la atmósfera y el espacio el flujo de calor reflejado por la tierra no pasa al espacio, quedándose en la atmósfera recalentándola. [3]

En condiciones normales, cuando la cantidad de gases de efecto invernadero no son excesivos la atmósfera terrestre constituye un ambiente propicio para la vida con temperatura alrededor de los $15^{\circ} \mathrm{C}$ y se dice que el efecto invernadero es positivo, pero cuando la cantidad de gases de efecto invernadero es excesiva la temperatura atmosférica aumenta significativamente y hace menos propicia la vida, conociéndose este estado como negativo o como de sobrecalentamiento global. Los gases de efecto invernadero (GEI), son aquellos que dejan pasar la radiación del sol, pero que absorben las radiaciones infrarrojas devueltas por la superficie terrestre y le impiden escapar hacia el espacio.

En la atmósfera existe una gran variedad de gases de efecto invernadero y los más perjudiciales son el vapor de agua $\left(\mathrm{H}_{2} \mathrm{O}\right)$, el dióxido de carbono $\left(\mathrm{CO}_{2}\right)$, el óxido nitroso $\left(\mathrm{N}_{2} \mathrm{O}\right)$, el metano $\left(\mathrm{CH}_{4}\right)$ y el ozono $\left(\mathrm{O}_{3}\right)$. [4] Para fines de la investigación se enfatizó el estudio en el dióxido de carbono $\left(\mathrm{CO}_{2}\right)$ y del metano $\left(\mathrm{CH}_{4}\right)$.

\subsection{CENTRALES HIDROELÉCTRICAS}

Una central hidroeléctrica es aquella que utiliza la energía potencial del agua almacenada para convertirla en energía eléctrica.

Principales componentes de las centrales hidroeléctricas:

- Turbina

- Generador

- Transformador de potencia

- Transformadores de medición de corriente

- Disyuntores

- Seccionadores

- Aisladores de paso

- Malla a tierra

\section{Clasificación de las centrales hidroeléctricas}

- Centrales de agua fluyente: Turbinan el agua disponible en el momento. Tienen el inconveniente de que, en época de sequía, no generan energía.

- Centrales con regulación propia (embalse): Se embalsa un volumen considerable de agua mediante la construcción de una o más presas que forman lagos artificiales; el embalse permite regular la cantidad de agua que pasa por las turbinas con el fin de unificar las variaciones temporales de los caudales afluentes en el río.

- Centrales a pie de presa: En este caso se coge un tramo de río o lago a partir del cual se represa el agua y las turbinas son colocadas justo a la salida de la represa. [5]

\subsection{EMBALSES DE LAS CENTRALES HIDROELECTRICAS}

Un embalse se forma como resultado de la acumulación de agua en una represa. El volumen que almacena depende de la altura de la presa y de la forma geométrica, definida por la topografía de la zona inundada, a la que también se le denomina "vaso". [6]

\subsection{EMISIONES DE GASES DE EFECTO INVERNADERO (GEI) EN EMBALSES DE CENTRALES HIDROELÉCTRICAS}

"Todos los sistemas de agua dulce, ya sean naturales o artificiales, emiten GEI debido a la descomposición de la materia orgánica que permanece sumergida. Los principales GEI emitidos son el dióxido de carbono ( $\left.\mathrm{CO}_{2}\right)$ y el metano $\left(\mathrm{CH}_{4}\right)$.

El dióxido de carbono se forma por la descomposición del carbono orgánico presente en el embalse. 
El metano es formado por bacterias metanogénicas y luego es consumido por la bacteria metanotrópica que descomponen la materia orgánica de las aguas y los sedimentos presentes en el fondo del embalse siendo nulo o de bajo contenido de oxígeno." [7]

Los GEI que se producen en Lima o en Londres, tienen consecuencias para todo el planeta. Es decir, el efecto es global más no local.

\subsection{EMBALSES \\ HIDROELÉCTRICOS PERUANOS SELECCIONADOS PARA LA INVESTIGACIÓN}

En el Perú existen muchos embalses hidroeléctricos, pero se ha seleccionado a los embalses que corresponden a centrales hidroeléctricas en funcionamiento y que tienen mayor generación de energía eléctrica en la actualidad, las cuales se describen a continuación:

\section{EMBALSE TABLACHACA (CENTRAL HIDROELÉCTRICA SANTIAGO ANTÚNEZ DE MAYOLO Y RESTITUCIÓN)}

Las centrales hidroeléctricas Santiago Antúnez de Mayolo y Restitución o comúnmente conocidas como el complejo hidroeléctrico del Mantaro, están ubicadas en la región de Huancavelica y han iniciado sus operaciones en 1973 y 1984 respectivamente. Ambas centrales se alimentan de la represa Tablachaca ubicada en la localidad de Quichuas, con capacidad de embalse de 8 millones de $\mathrm{m}^{3}$, en la que se almacena el agua proveniente del rio Mantaro. Es la generadora hidroeléctrica más grande del país en la actualidad, contribuyendo con $1.008 \mathrm{MW}$.

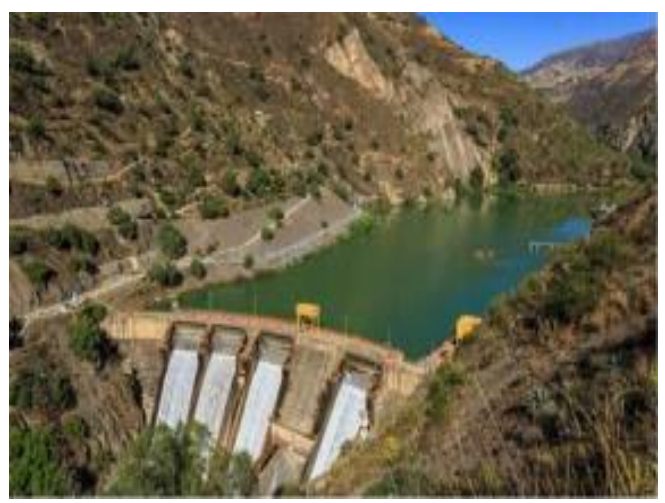

Fig. 1. Embalse de Tablachaca [8]

\section{EMBALSE CERRO DEL ÁGUILA (CENTRAL HIDROELÉCTRICA CERRO DEL ÁGUILA)}

La central hidroeléctrica Cerro del águila, está ubicada en la provincia de Tayacaja, región de Huancavelica; inició sus operaciones el 2016 y aprovecha las aguas del río Mantaro. El embalse Cerro del águila tiene una capacidad de almacenamiento de 370 DOI: https://doi.org/10.21754/tecnia. 10.21754/tecnia.v21i2.86 millones de $\mathrm{m}^{3}$ aproximadamente, es la segunda generadora hidroeléctrica más grande del país, contribuyendo con 513 MW de energía eléctrica.

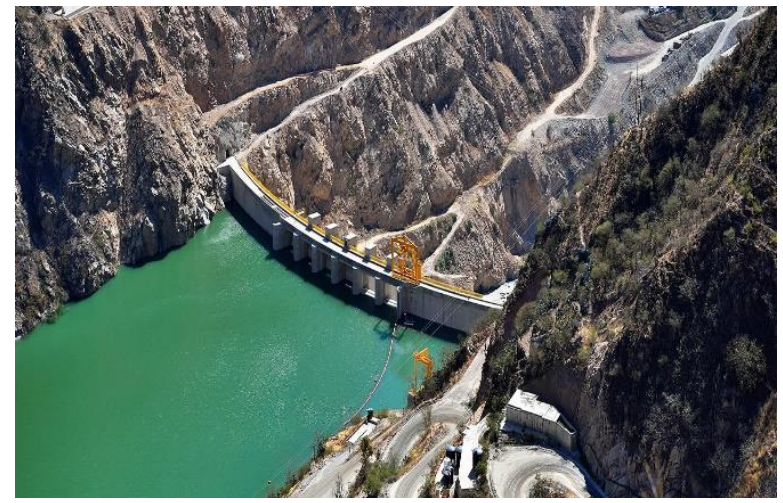

Fig. 2. Embalse Cerro del águila[9]

\section{EMBALSE CHAGLLA (CENTRAL HIDROELÉCTRICA CHAGLLA)}

La central hidroeléctrica Chaglla, está ubicada en la provincia de Huánuco y Pachitea, región de Huánuco; inició sus operaciones en el 2016 y aprovecha las aguas del río Huallaga. Cuenta con una potencia instalada de 513 MW, en el año 2017 tuvo una producción de 2095 GWh.

La represa (embalse Chaglla) tiene una capacidad de almacenamiento de 356 millones de $\mathrm{m}^{3}$ por la que es la segunda más alta a nivel mundial.

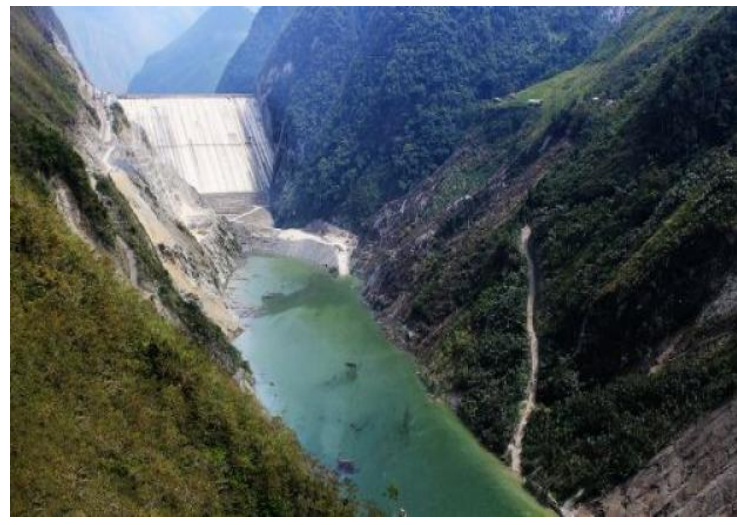

Fig. 3. embalse Chaglla[10]

\section{EMBALSE SHEQUE (CENTRAL HIDROELÉCTRICA HUINCO)}

La central hidroeléctrica Huinco, está ubicada en el distrito de San Pedro de Casta, en la provincia de Huarochirí, departamento de Lima; inició sus operaciones en el año 1964; recibe aguas del río Santa Eulalia y de la cuenca Marcapomacocha, tiene una potencia instalada de $168.5 \mathrm{MW}$. 


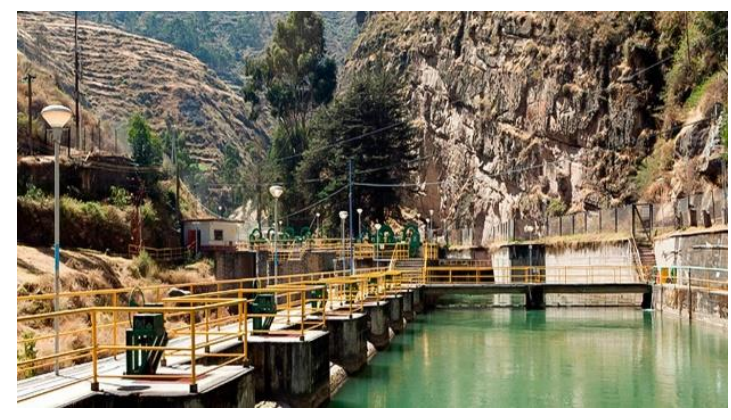

Fig. 4. Embalse Sheque.[11]

\section{EMBALSE CAPILLUCAS (CENTRAL HIDROELÉCTRICA EL PLATANAL)}

Genera una potencia de $220 \mathrm{MW}$ y opera desde el año 2010 y se ubica en la cuenca del río Cañete, en el distrito de Zúñiga, en las provincias de Yauyos y Cañete. Capta las aguas de la laguna Paucarcocha a 4,200 msnm, en el distrito de Tanta, provincia de Yauyos, la cual ha sido embalsada mediante un muro de contención de 28 metros de alto para elevar su capacidad hasta los 70 millones de $\mathrm{m}^{3}$.

Recorre 100 kilómetros río abajo hasta la represa de regulación de Capillucas con capacidad de 1,8 millones de $\mathrm{m}^{3}$. En el año 2017 permitió generar 1183.7 GWh, la cual se condujo hasta la subestación de Chilca, en la costa de Cañete, mediante una línea de transmisión de 220 kilovoltios (KV), alimentando así al sistema interconectado nacional.

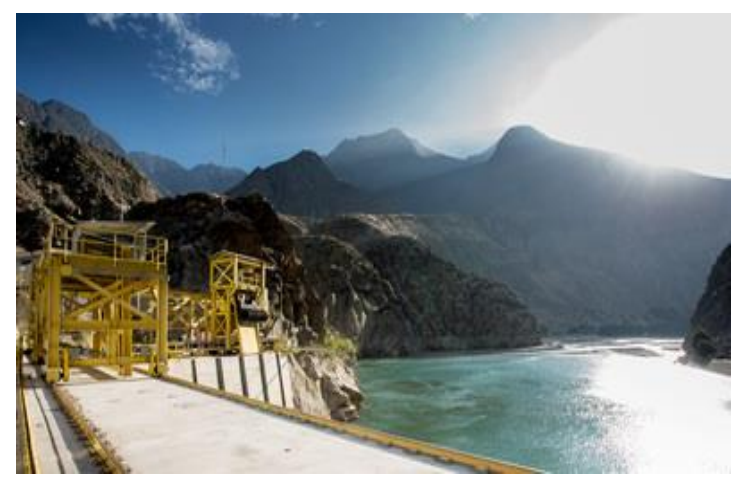

Fig. 6. Embalse Capillucas.[12]

\section{EMBALSE CHECRAS (CENTRAL HIDROELÉCTRICA CHEVES)}

La central hidroeléctrica Cheves está ubicada en las provincias de Huaura y Oyón, departamento de Lima; inició sus operaciones en el 2015, utiliza parte de las aguas de los ríos Huaura y Checras. El embalse Checras tiene una capacidad de almacenamiento de $620 \mathrm{mil} \mathrm{m}^{3}$. La capacidad es de $168 \mathrm{MW}$ y la energía anual generada en el 2017 fue de 851.5 GWh.

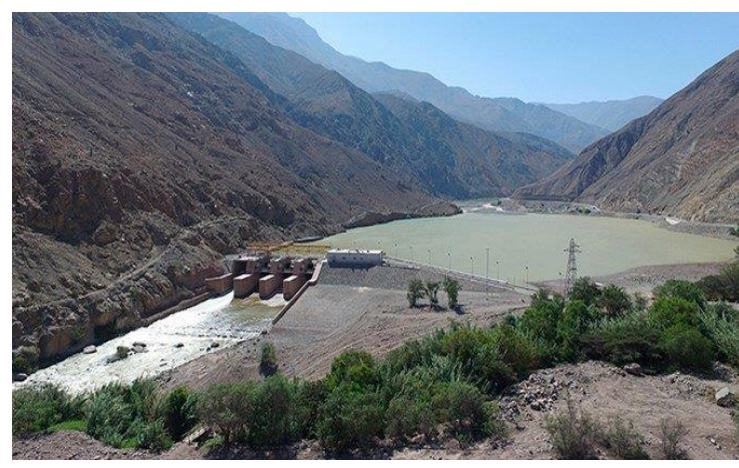

Fig. 5. Embalse Checras.[13]

\section{EMBALSE AGUADA BLANCA (CENTRAL HIDROELÉCTRICA CHARCANI V)}

Inaugurada en 1989. Se encuentra en el distrito de Alto Selva Alegre, provincia y departamento de Arequipa. Construida cerca al volcán Misti. La central aprovecha las aguas del río chili, tomadas del embalse Aguada Blanca que tiene una capacidad de 43 millones de $\mathrm{m}^{3}$. Su casa de máquinas en caverna cuenta con tres turbinas Pelton, las cuales generan una potencia de 153 MW en total.

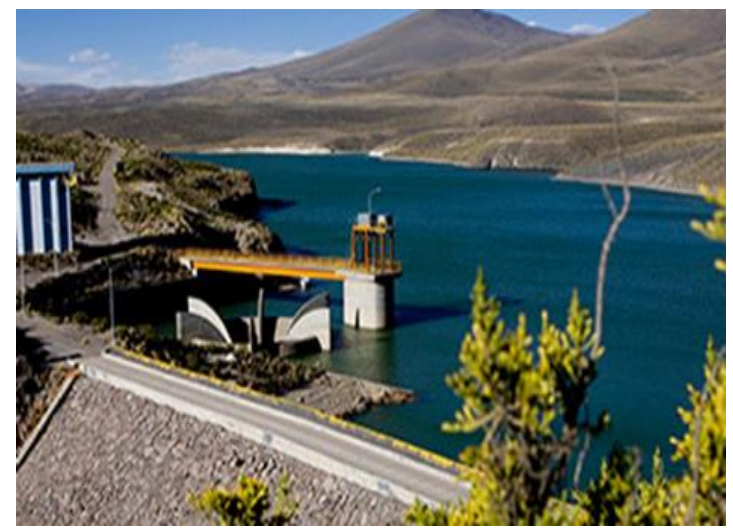

Fig. 7. Embalse Aguada Blanca.[14]

\section{EMBALSE TULUMAYO HIDROELÉCTRICA CHIMAY)}

La central hidroeléctrica Chimay, está ubicada en el distrito de Monobamba, provincia de Jauja, departamento de Junín; inició sus operaciones en el 2000.

La central toma las aguas del río Tulumayo con un embalse del mismo nombre, que tiene una capacidad de 2,1 millones de $\mathrm{m}^{3}$ y su potencia instalada es de $152 \mathrm{MW}$. 


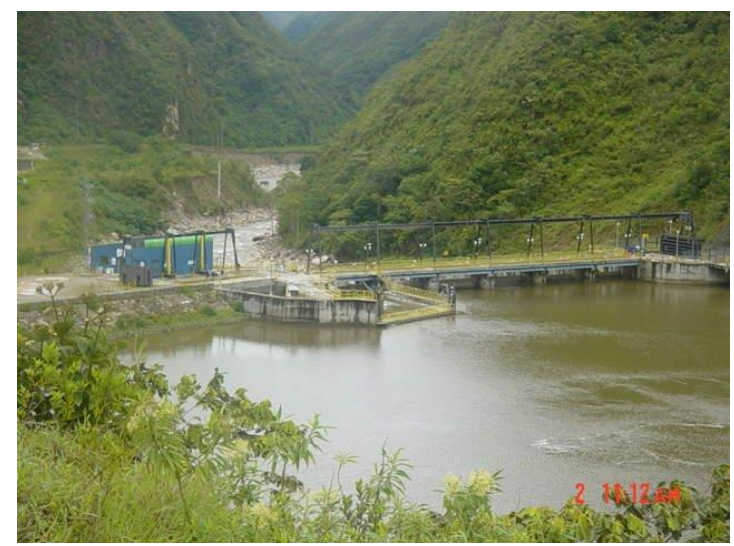

Fig. 8. Embalse Tulumayo.[15]

\section{Embalse Yuracmayo (central hidroeléctrica Matucana)}

La central hidroeléctrica Matucana también llamada Pablo Boner en alusión a su gestor, se encuentra ubicada en el distrito de San Jerónimo de Surco, provincia de Huarochirí, departamento de Lima, comenzó a funcionar en 1972.

Esta central utiliza las aguas del río Rímac y el embalse Yuracmayo que tiene una capacidad de 48 millones de $\mathrm{m}^{3}$, mediante una captación denominada toma Tamboraque y cuenta con dos grupos de generación con turbinas Pelton de eje horizontal, acoplados a generadores síncronos que generan una potencia instalada de $140 \mathrm{MW}$.

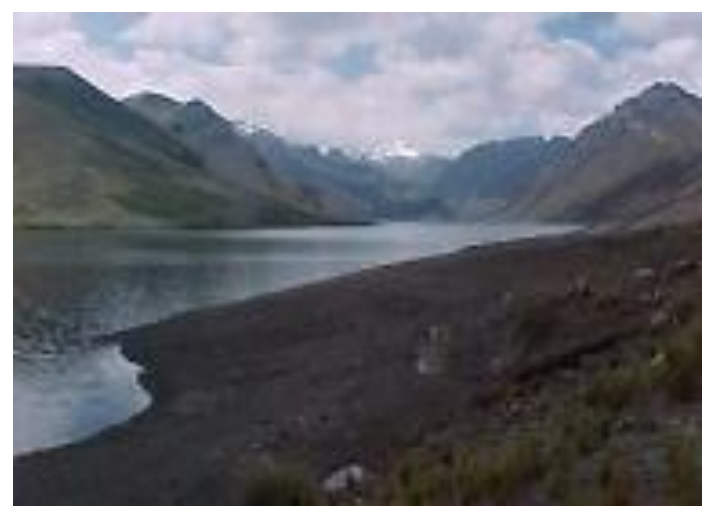

Fig. 9. embalse Yuracmayo.

\section{Embalse Huallamayo (central hidroeléctrica Yuncán)}

La central hidroeléctrica Yuncán inicio sus operaciones en 2005, se encuentra localizada en el departamento de Pasco, entre las cuencas de los ríos Paucartambo y Huachón a aproximadamente 2,500 msnm. Consta de tres turbinas pelton que generan en total $134 \mathrm{MW}$.
La central está conformada por la presa Uchuhuerta y la presa Huallamayo y esta última puede almacenar hasta 1.6 millones de $\mathrm{m}^{3}$ de agua.

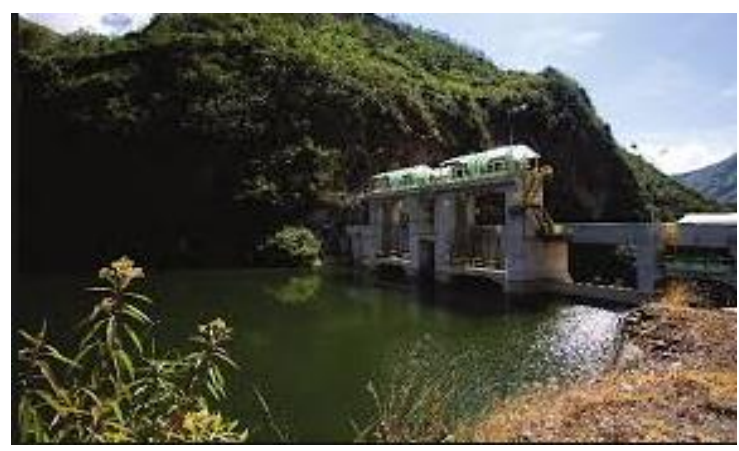

Fig. 10. embalse Huallamayo.[16]

\section{Embalse Itaipú (central hidroeléctrica Itaipú} binacional)

La central inició sus operaciones en 1984 y está ubicada en el río Paraná en la frontera entre Paraguay y Brasil, su embalse Itaipú tiene una capacidad de 29 billones de $\mathrm{m}^{3}$ y un área inundada de $1350 \mathrm{Km}^{2}$.

Cuenta con una potencia instalada de $14000 \mathrm{MW}$ obteniendo así actualmente el segundo lugar como la central más grande del mundo, pero ocupa el primer lugar como la generadora más grande de energía eléctrica limpia y renovable en el planeta.

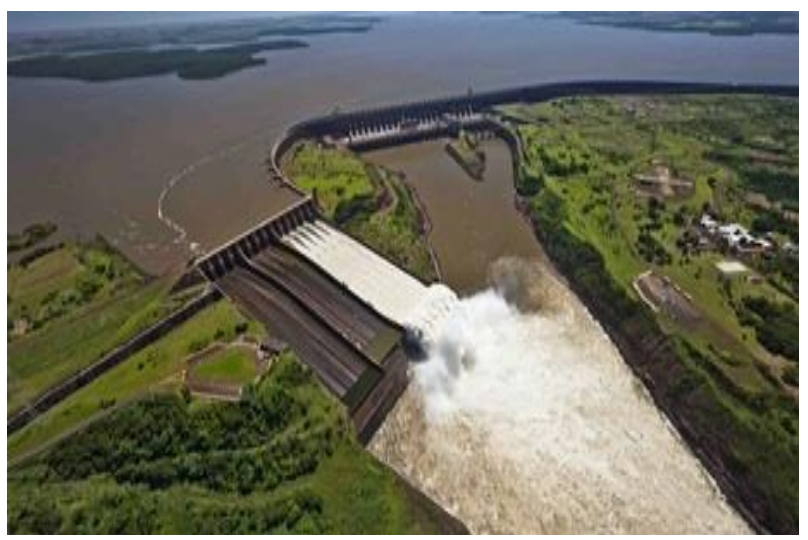

Fig.11. embalse de Itaipú.[17]

\section{METODO DEL IPCC -2006 PARA DETERMINAR LAS EMISIONES DE $\mathrm{CO}_{2} \mathrm{Y} \mathrm{CH}_{4}$}

Fórmula para determinar las emisiones de $\mathrm{CO}_{2}$

Para determinar las emisiones difusoras de Dióxido de Carbono $\left(\mathrm{CO}_{2}\right)$ en los reservorios se optó por utilizar el método del panel intergubernamental para el cambio climático propuesto por el IPCC -2006, apéndice 2, Nivel 2. [18] 
El cual contempla la siguiente ecuación 1:

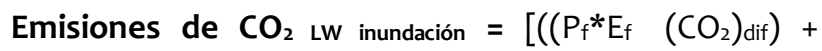
$\left.\left.\left(P_{i}^{*} E_{i}\left(C O_{2}\right)_{\text {dif }}\right)\right) *\left(A_{\text {inundación, superficie }} * f_{A} * 10^{-6}\right)\right]$

Dónde:

Emisiones de $\mathrm{CO}_{2}$ LWinundación $=$ Total de las emisiones de $\mathrm{CO}_{2}$ provenientes de las tierras convertidas en tierras inundadas expresado en $\mathrm{Gg}$ de $\mathrm{CO}_{2}$ año-1.

$\mathbf{P}_{\mathbf{f}}=$ Periodo libre de hielos expresado en días año-1 $\mathbf{P}_{\mathbf{i}}=$ Periodo cubierto de hielos, días año-1 (los períodos cubiertos de hielos no son aplicables para nuestra región).
$\mathbf{E}_{\mathbf{f}}\left(\mathrm{CO}_{2}\right)_{\text {dif }}=$ Promedio diario de las emisiones difusoras provenientes de la interfaz aire-agua durante el periodo libre de hielos expresado en $\mathrm{kg}$ de $\mathrm{CO}_{2} \mathrm{ha}^{-1} \mathrm{dia}^{-1}$.

$\mathbf{E}_{\mathbf{i}}\left(\mathrm{CO}_{2}\right)_{\text {dif }}=$ Emisiones difusoras relacionadas con el periodo cubierto de hielos expresado en $\mathrm{kg}$ de $\mathrm{CO}_{2} \mathrm{ha}^{-1}$ $\mathrm{dia}^{-1}$.

A inundación, superficie total = Área total de la superficie del reservorio, incluidas las tierras inundadas, los lagos y ríos expresado en ha.

$\mathbf{f}_{\mathrm{A}}=$ Fracción del área total del reservorio que se inundó en los últimos 10 años, adimensional.

TABLA I

Emisiones medidas de $\mathrm{CO}_{2}$ provenientes de tierras inundadas (IPCC, 2006). [18]

\begin{tabular}{|c|c|c|c|c|c|c|}
\hline \multirow[t]{3}{*}{ Clima } & \multirow{2}{*}{\multicolumn{5}{|c|}{$\begin{array}{l}\text { Emisiones difusoras (periodo libre de hielos) } \\
\mathrm{E}_{\mathrm{f}}\left(\mathrm{CO}_{2}\right)_{\mathrm{dif}}\left(\mathrm{Kg} \cdot \text { de } \mathrm{CO}_{2} \mathrm{ha}^{-1} \mathrm{día}^{-1}\right)\end{array}$}} & \multirow{3}{*}{ Referencias } \\
\hline & & & & & & \\
\hline & Mediana & \multirow{2}{*}{ Min } & \multirow{2}{*}{ Máx } & \multirow{2}{*}{$\begin{array}{l}N_{m} \\
1011\end{array}$} & $\mathrm{~N}_{\text {res }}$ & \\
\hline $\begin{array}{l}\text { Polar / boreal muy } \\
\text { húmedo }\end{array}$ & 11.8 & & & & 20 & Bergström et al., 2004; Åberg et al., 2004; Huttunen et al., 2002 \\
\hline $\begin{array}{l}\text { Templado frío, } \\
\text { húmedo }\end{array}$ & 15.2 & 4.5 & 86.3 & 633 & 20 & $\begin{array}{l}\text { Duchemin, 2000; Schlellhase et al., } 1994 \text {; Duchemin et al., } 1999 \text {; } \\
\text { Duchemin et al., 1995; Tremblay et al., } 2005\end{array}$ \\
\hline $\begin{array}{l}\text { Templado cálido, } \\
\text { húmedo }\end{array}$ & 8.1 & -10.3 & 57.5 & 507 & 33 & $\begin{array}{l}\text { Duchemin, 2000; Duchemin, 2002a ; St-Louis et al., 2000; Smith y } \\
\text { Lewis, } 1992 \text {; Tremblay et al., } 2005\end{array}$ \\
\hline $\begin{array}{l}\text { Templado cálido, } \\
\text { seco }\end{array}$ & 5.2 & -12.0 & 31.0 & 390 & 43 & Soumis et al., 2004 ; Therrien et al., 2005 \\
\hline $\begin{array}{l}\text { Tropical, muy } \\
\text { húmedo }\end{array}$ & 44.9 & 11.5 & 90.9 & 642 & 7 & $\begin{array}{l}\text { Keller y Stallard, 1994; Galy-Lacaux et al., 1997; Galy-Lacaux, 1996; } \\
\text { Duchemin et al., 2000; Pinguelli Rosa et al., 2002; Tavares de lima } \\
\text { et al., 2002; Tavares de lima, } 2005\end{array}$ \\
\hline Tropical, seco & 39.1 & 11.7 & 58.7 & 197 & 5 & Pinguelli Rosa et al., 2002; Dos Santos, 2000 \\
\hline
\end{tabular}

Nota. Los valores de la segunda columna representan las medianas de las emisiones de $\mathrm{CO}_{2}$ declaradas en la bibliografía, las cuales corresponden en sí a las medias aritméticas de los flujos medidos sobre los reservorios individuales. Se usan las medianas pues las distribuciones de frecuencia de las mediciones de flujo subyacentes no son normales y sus medias aritméticas están ya sesgadas por los valores extremos. Los valores Mín y Máx. corresponden a los más bajos y los más altos de todas las mediciones individuales dentro de una región climática dada; se dan sólo como indicación de la variabilidad. $\mathrm{N}_{\mathrm{m}}$ = cantidad de mediciones; $\mathrm{N}_{\text {res }}=$ cantidad de reservorios muestreados. Estás mediciones pueden incluir las emisiones no antropogénicas (p. ej., emisiones provenientes del carbono en la cuenca corriente arriba) y posibles cómputos dobles de emisiones antropogénicas (p. ej., aguas servidas de las zonas urbanas de la región del reservorio) y de este modo pueden sobreestimar las emisiones. 
Para estimar el promedio diario de las emisiones difusoras de $\mathrm{CO}_{2}$ se debe aplicar los valores establecidos en la tabla 1, en la que se visualizan las emisiones medidas para diversas zonas climáticas teniendo en cuenta las variaciones espaciales intra reservorios o regionales y variaciones temporales estacionales establecidas por las emisiones de los reservorios y los flujos en la interfaz agua.

\section{Fórmula para determinar las emisiones de $\mathrm{CH}_{4}$}

Para determinar las emisiones difusoras de Metano $\left(\mathrm{CH}_{4}\right)$ en los reservorios se optará por utilizar el método del panel intergubernamental para el cambio climático propuesto por el IPCC -2006, apéndice 3, Nivel 1. El cual contempla la siguiente ecuación 2. [19]:

Emisiones de $\mathrm{CH}_{4} \mathrm{ww}$ inundación $=\mathrm{P} * \mathrm{E}\left(\mathrm{CH}_{4}\right)$ dif ${ }^{*} \mathrm{~A}$ inundación, superficie total $* 10^{-6}$

\section{Dónde:}

Emisiones de $\mathrm{CH}_{4}$ wwinundación = Total de las emisiones de $\mathrm{CH}_{4}$ provenientes de las tierras inundadas expresado en $\mathrm{CH}_{4}$ año-1.

$\mathbf{P}=$ periodo libre de hielos, días año-1 (por lo general, 365 para las estimaciones de inventario anuales, o menos en los países con periodo con cobertura de hielo).
$\mathbf{E}\left(\mathrm{CH}_{4}\right)_{\text {dif }}=$ promedio diario de las emisiones difusoras, expresado en $\mathrm{kg}$. De $\mathrm{CH}_{4} \mathrm{ha}^{-1} \mathrm{dia}^{-1}$.

A inundación superficie total = área total de la superficie inundada, incluidas las tierras inundadas, los lagos y ríos, expresado en ha.

Para estimar el promedio diario de las emisiones difusoras de $\mathrm{CH}_{4}$ se debe aplicar los valores establecidos en la tabla 2, donde se visualizan las emisiones medidas para diversas zonas climáticas, integrando las variaciones espaciales intra reservorios o regionales y variaciones temporales estacionales establecidos por las emisiones de los reservorios, de igual forma los flujos en la interfaz agua aire.

En la tabla 3 se puede observar los valores calculados de las áreas inundadas y el clima seleccionado según las características del lugar de ubicación de cada central hidroeléctrica.

TABLA II

Emisiones medidas de $\mathrm{CH}_{4}$ proveniente de las tierras inundadas.[19]

\begin{tabular}{|c|c|c|c|c|c|c|}
\hline \multirow[t]{2}{*}{ Clima } & \multicolumn{5}{|c|}{$\begin{array}{l}\text { Emisiones difusoras (periodo libre de hielos) } \\
\mathrm{E}_{\mathrm{f}}\left(\mathrm{CH}_{4}\right)_{\text {dif }}\left(\mathrm{Kg} \text {. de } \mathrm{CH}_{4} \mathrm{ha}^{-1} \mathrm{dí}^{-1}\right)\end{array}$} & \multirow[t]{2}{*}{ Referencias } \\
\hline & Mediana & Min. & Máx. & $\mathbf{N}_{\mathrm{m}}$ & $\mathbf{N}_{\text {res }}$ & \\
\hline $\begin{array}{l}\text { Polar / boreal } \\
\text { muy húmedo }\end{array}$ & 0.086 & 0.011 & 0.3 & 253 & 13 & $\begin{array}{l}\text { Blais 2005; Tremblay et al, 2005; Therrien, 2004; Therrien, 2005; } \\
\text { Huttunen et al., 2002; Lambert, 2002; Duchemin, } 2000\end{array}$ \\
\hline $\begin{array}{l}\text { Templado frío, } \\
\text { húmedo }\end{array}$ & 0.061 & 0.001 & 0.2 & 233 & 10 & $\begin{array}{l}\text { Tremblay et al., 2005; Therrien, 2004; Blais, 2005; Lambert, 2002; } \\
\text { Duchemin et al., } 1999\end{array}$ \\
\hline $\begin{array}{l}\text { Templado cálido, } \\
\text { húmedo }\end{array}$ & 0.150 & -0.05 & 1.1 & 416 & 16 & $\begin{array}{l}\text { Tremblay et al., 2005; Soumis et al., 2004; Duchemin, 2000; Smith y } \\
\text { Lewis, } 1992\end{array}$ \\
\hline $\begin{array}{l}\text { Templado cálido, } \\
\text { seco }\end{array}$ & 0.044 & 0.032 & 0.09 & 135 & 5 & Therrien et al., 2005; Therrien, 2004; Soumis et al., 2004 \\
\hline $\begin{array}{l}\text { Tropical, muy } \\
\text { húmedo }\end{array}$ & 0.630 & 0.067 & 1.3 & 303 & 6 & $\begin{array}{l}\text { Tavares de lima, 2005; Abril et al., 2005; Therrien, 2004; Rosa et al., 2002; } \\
\text { Tavares de lima et al., 2002; Duchemin et al., 2000; Galy-Lacaux et al., } \\
\text { 1997; Galy-Lacaux, 1996; Keller y Stallard,1994 }\end{array}$ \\
\hline
\end{tabular}

$\begin{array}{llllll}\text { Tropical, seco } & 0.295 & 0.070 & 1.1 & 230 & 5\end{array}$ Rosa et al., 2002; Dos Santos, 2000

Nota. Los valores de la segunda columna representan las medianas de las emisiones de $\mathrm{CH}_{4}$ declaradas en la bibliografía, las cuales corresponden, en sí, a las medias aritméticas de los flujos medidos sobre los reservorios individuales. Se usan las medianas, pues la frecuencia de las distribuciones de las mediciones de flujo subyacentes no es normal y sus medias aritméticas están ya sesgadas por los valores extremos. Los valores Mín. y Máx. Corresponden a los más bajos y los más altos de todas las mediciones individuales dentro de una región climática dada; se dan sólo como una indicación de la variabilidad. $\mathrm{N}_{\mathrm{m}}=$ cantidad de mediciones; $\mathrm{N}_{\text {res }}=$ cantidad de reservorios muestreados. Estas mediciones pueden incluir las emisiones no antropogénicas (p. ej., emisiones provenientes del carbono en la cuenca corriente arriba) y posibles cómputos dobles de emisiones antropogénicas (p. ej., aguas servidas de las zonas urbanas de la región del reservorio) y de este modo pueden sobreestimar las emisiones. 
TABLA III

Área inundada de las principales centrales hidroeléctricas del Perú

\begin{tabular}{|c|c|c|c|c|c|c|}
\hline Hidroeléctrica & Departamento & $\begin{array}{l}\text { Área } \\
\text { inundada } \\
\text { (ha) }\end{array}$ & $\begin{array}{l}\text { Año puesta } \\
\text { en servicio }\end{array}$ & Clima & $\begin{array}{l}\text { Capacidad } \\
\text { instalada (MW) }\end{array}$ & $\begin{array}{l}\text { Fracción del área } \\
\text { total del reservorio } \\
\text { que se inundó en los } \\
\text { últimos } 10 \text { años. fA }\end{array}$ \\
\hline $\begin{array}{l}\text { Complejo del } \\
\text { Mantaro }\end{array}$ & Huancavelica & 9642.86 & 1973 & $\begin{array}{l}\text { Templado frío, } \\
\text { húmedo }\end{array}$ & 1000 & 0.7 \\
\hline Cerro del águila & Huancavelica & 4946.79 & 2016 & $\begin{array}{l}\text { Templado frío, } \\
\text { húmedo }\end{array}$ & 513 & 0.4 \\
\hline Chaglla & Huánuco & 4397.14 & 2016 & $\begin{array}{l}\text { Templado, frio } \\
\text { húmedo }\end{array}$ & 456 & 0.4 \\
\hline Huinco & Lima & 2491.71 & 1964 & $\begin{array}{l}\text { Templado, frio } \\
\text { húmedo }\end{array}$ & 258.4 & 0.5 \\
\hline El Platanal & Lima & 2121.43 & 2010 & $\begin{array}{l}\text { Templado cálido, } \\
\text { húmedo }\end{array}$ & 220 & 0.7 \\
\hline Cheves & Lima & 1621.93 & 2015 & $\begin{array}{l}\text { Templado cálido, } \\
\text { húmedo }\end{array}$ & 168.2 & 0.7 \\
\hline Charcani V & Arequipa & $1475 \cdot 36$ & 1988 & $\begin{array}{l}\text { Templado cálido, } \\
\text { húmedo }\end{array}$ & 153 & 0.6 \\
\hline Chimay & Junín & 1465.71 & 2000 & $\begin{array}{l}\text { Templado, frio } \\
\text { húmedo }\end{array}$ & 152 & 0.7 \\
\hline Matucana & Lima & 1350.00 & 1972 & $\begin{array}{l}\text { Templado cálido, } \\
\text { húmedo }\end{array}$ & 140 & 0.7 \\
\hline Yuncan & Pasco & 1292.14 & 2005 & $\begin{array}{l}\text { Templado, frio } \\
\text { húmedo }\end{array}$ & 134 & 0.4 \\
\hline
\end{tabular}

\section{CALCULO DE LAS EMISIONES DIFUSORAS DE $\mathrm{CO}_{2} \mathrm{Y}$}

$\mathrm{CH}_{4}$

Se debe mencionar que los periodos cubiertos de hielo no son aplicables para nuestra región por lo tanto $\mathrm{Pi}_{\mathrm{y}} \mathrm{E}_{\mathrm{i}}\left(\mathrm{CO}_{2}\right)$ son iguales a o (cero).

El valor de $P_{f}$ es igual a 365 días, el resto de datos aplicados en las ecuaciones 1 y 2 se obtienen de las tablas 1, 2 y 3 según la característica de los embalses.
Por medio de los cálculos realizados en los embalses de las centrales hídricas peruanas se estimaron valores para las emisiones difusoras de $\mathrm{CO}_{2} \mathrm{y}$ $\mathrm{CH}_{4}$, obteniendo resultados de $83.947 \mathrm{Gg}_{\text {año }}{ }^{-1}$ ó 83947 Ton $\mathrm{CO}_{2}$ año-1 para las emisiones difusoras de $\mathrm{CO}_{2}$ y de $0.870 \mathrm{Gg}$ año ${ }^{-1}$ ó 870 Ton $\mathrm{CH}_{4}$ año ${ }^{-1}$ para las emisiones difusoras de $\mathrm{CH}_{4}$, tal como se representa en la figura 12.

TABLA IV

Emisiones difusoras de $\mathrm{CO}_{2}$ y $\mathrm{CH}_{4}$ de los embalses de las principales centrales hidroeléctricas del Perú

$\begin{array}{ccccc}\text { Hidroeléctrica } & \text { Área inundada } & \text { Emisiones de } \mathrm{CO}_{2}(\mathrm{Gg} & \begin{array}{c}\text { Emisiones de } \\ \mathrm{CH}_{4}(\mathrm{Gg} \text { año-1) }\end{array} & \begin{array}{c}\text { Emisiones de } \mathrm{CH}_{4} \text { convertidas } \\ \text { en } \mathrm{CO}_{2-\mathrm{Eq}}\end{array}\end{array}$

\begin{tabular}{|c|c|c|c|c|c|}
\hline Tablachaca & 9642.86 & 37.449 & 0.215 & 5.367 & 42.816 \\
\hline $\begin{array}{l}\text { Cerro del } \\
\text { águila }\end{array}$ & 4946.79 & 10.978 & 0.11 & 2.754 & 13.731 \\
\hline Chaglla & 4397.14 & 9.758 & 0.098 & 2.448 & 12.206 \\
\hline Sheque & 2491.71 & 6.912 & 0.055 & 1.387 & 8.299 \\
\hline Capillucas & 2121.43 & 4.39 & 0.116 & 2.904 & 7.294 \\
\hline Checras & 1621.93 & 3.357 & 0.089 & 2.22 & 5.577 \\
\hline $\begin{array}{l}\text { Aguada } \\
\text { blanca }\end{array}$ & 1475.36 & 2.617 & 0.081 & 2.019 & 4.637 \\
\hline Tulumayo & 1465.71 & 5.692 & 0.033 & 0.816 & 6.508 \\
\hline Yuracmayo & 1350 & 2.794 & 0.074 & 1.848 & 4.642 \\
\hline Huallamayo & 1292.14 & 2.87 & 0.03 & 0.719 & 3.587 \\
\hline TOTAL & 30805.07 & 83.947 & 0.87 & 21.762 & 105.71 \\
\hline
\end{tabular}




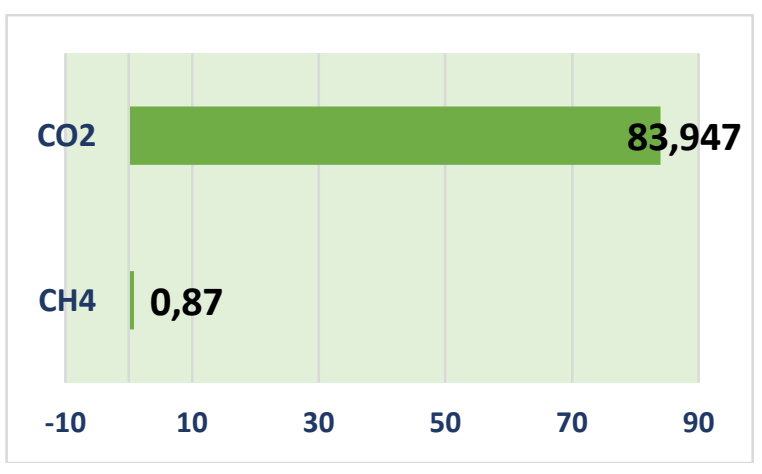

Fig. 3: emisiones de $\mathrm{CO}_{2}$ y $\mathrm{CH}_{4}(\mathrm{Gg}$ año-1)

\section{COMPARACIÓN DE LAS EMISIONES ESTIMADAS DE $\mathrm{CO}_{2} \mathrm{Y} \mathrm{CH}_{4}$ CON LAS EMISIONES DE LAS CENTRALES DE BRASIL}

Se realiza una comparación de las emisiones de los embalses peruanos analizados con embalses brasileños, se tomó para esta comparación las emisiones estimadas de los embalses de las siguientes centrales hidroeléctricas brasileñas: Tres Marías, Barra Bonita, Tucurui, Samuel, Itaipú, Segredo, Xingó, Miranda, Sobradinho y Serra da mesa, cuya información se tomó de la publicación "GHG emissions from hydroelectric reservoirs in tropical and equatorial regions: Review of 20 years of $\mathrm{CH}_{4}$ emission measurements",[20] como se puede apreciar en la tabla 5 .

TABLA V

Emisiones de $\mathrm{CO}_{2}$ y $\mathrm{CH}_{4}$ de los embalses de las principales centrales hidroeléctricas de Brasi

\begin{tabular}{|c|c|c|c|c|c|}
\hline Embalses & $\begin{array}{l}\text { Área } \\
\text { inundada (ha) }\end{array}$ & $\begin{array}{l}\text { Emisiones } \mathrm{CO}_{2} \\
\text { (Gg año-1) }\end{array}$ & $\begin{array}{l}\text { Emisiones } \mathrm{CH}_{4} \text { (Gg } \\
\text { año-1) }\end{array}$ & $\begin{array}{l}\text { Emisiones de } \mathrm{CH}_{4} \\
\text { convertidas en } \mathrm{CO}_{2-\mathrm{Eq}}(\mathrm{Gg} \\
\text { año-1) }\end{array}$ & $\begin{array}{l}\text { Emisiones totales } \\
\mathrm{CO} 2-\mathrm{Eq} \text { (Gg año-1) }\end{array}$ \\
\hline
\end{tabular}

\begin{tabular}{|c|c|c|c|c|c|}
\hline Tres marías & 115500 & 424.114 & 74.508 & 1862.7 & 2286.814 \\
\hline Barra bonita & 33431 & 453.88 & 2.379 & 59.475 & 513.355 \\
\hline Tucurui & 287500 & 7516.551 & 97.001 & 2425.025 & 9941.576 \\
\hline Samuel & 56000 & $1519 \cdot 559$ & 21.224 & 530.6 & 2050.159 \\
\hline Itaipú & 135000 & 48.34 & 5.88 & 147 & $195 \cdot 34$ \\
\hline Segredo & 8200 & 80.663 & 0.263 & 6.575 & 87.238 \\
\hline Xingó & 6000 & 134.434 & 0.878 & 21.95 & 156.384 \\
\hline Miranda & 7000 & 81.046 & 2.847 & 71.175 & 152.221 \\
\hline Serra da mesa & 178400 & 1293.529 & 16.637 & 415.925 & 1709.454 \\
\hline Sobradinho & 4214 & 40.56 & 0.436 & 36.78 & 154.35 \\
\hline TOTAL & 831245 & 11592.676 & 222.053 & 5577.205 & 17246.891 \\
\hline
\end{tabular}

Se puede apreciar que Brasil tiene centrales hidroeléctricas mucho más grandes que el Perú.

En la tabla 6 presentaremos el factor de los embalses peruanos y en la tabla 7 los embalses brasileños.
Coeficiente Ton de $\mathrm{CO} / \mathrm{KW}$ en centrales peruanas

\begin{tabular}{lrrr}
\hline Embalse & $\begin{array}{l}\text { Capacidad } \\
\text { instalada } \\
(\mathrm{MW})\end{array}$ & $\begin{array}{l}\text { Emisiones de } \\
\mathrm{CO}_{2} \text { (Gg año-1) }\end{array}$ & $\begin{array}{l}\text { Emision CO2 } \\
\text { / Capacidad } \\
\text { (ton/KW) }\end{array}$ \\
\hline Tablachaca & 1000 & 37.449 & 0.037449 \\
\hline Cerro del águila & 513 & 10.978 & 0.02139961 \\
\hline Chaglla & 456 & 9.758 & 0.02139912 \\
\hline Huinco & 258.4 & 6.912 & 0.02674923 \\
\hline El Platanal & 220 & 4.39 & 0.01995455 \\
\hline Cheves & 168.2 & 3.357 & 0.01995838 \\
\hline Charcani V & 153 & 2.617 & 0.01710458 \\
\hline Chimay & 140 & 5.692 & 0.03744737 \\
\hline Matucana & 134 & 2.794 & 0.01995714 \\
\hline Yuncan & 3194.6 & 86.817 & 0.027176172 \\
\hline TOTAL & & & 0.02141791 \\
\hline
\end{tabular}


TABLA VII

Coeficiente Ton de $\mathrm{CO}_{2} / \mathrm{KW}$ en centrales brasileñas

\begin{tabular}{lrrr}
\hline Embalse & $\begin{array}{l}\text { Capacidad } \\
\text { instalada } \\
(\mathrm{MW})\end{array}$ & $\begin{array}{l}\text { Emisiones } \\
\text { de } \mathrm{CO}_{2}(\mathrm{Gg} \\
\text { año-1 })\end{array}$ & $\begin{array}{l}\text { Emision CO2 / } \\
\text { Capacidad } \\
\text { (ton/KW) }\end{array}$ \\
\hline Tres marías & 396 & 424.114 & 1.070994949 \\
\hline Barra bonita & 140 & 453.88 & 3.242 \\
\hline Tucurui & 8370 & 7516.551 & 0.898034767 \\
\hline Samuel & 216 & 1519.559 & 7.03499537 \\
\hline Itaipú & 14000 & 48.34 & 0.003452857 \\
\hline Segredo & 1260 & 80.663 & 0.064018254 \\
\hline Xingó & 3162 & 134.434 & 0.042515497 \\
\hline Miranda & 390 & 81.046 & 0.207810256 \\
\hline Serra da mesa & 855 & 1293.529 & 1.512899415 \\
\hline Sobradinho & 1050 & 40.56 & 0.03862857 \\
\hline TOTAL & 29839 & 11592.676 & 0.38850752 \\
\hline
\end{tabular}

Se puede observar el caso del embalse Tablachaca Perú que tiene similar área inundada que el embalse de Segredo - Brasil, pero este último tiene casi el doble de emisiones totales $\mathrm{CO}_{2-\mathrm{Eq}}$ que el embalse de Tablachaca.

Brasil tiene un clima tropical, lo cual no le ayuda en la disminución de las emisiones de $\mathrm{CO}_{2}$ y $\mathrm{CH}_{4}$, por el contrario, el Perú es afortunado en tener un clima y temperaturas variables porque eso nos permite tener menor emisión de $\mathrm{CO}_{2}$ y $\mathrm{CH}_{4}$ en nuestros embalses

\section{CONCLUSIONES Y RECOMENDACIONES}

- Las emisiones de $\mathrm{CO}_{2}$ y $\mathrm{CH}_{4}$ calculadas con la metodología del IPCC para el presente estudio, dan una idea general de la situación de los embalses analizados. Se pudo estimar que los embalses aportan una cantidad de 105.71 ( $\mathrm{Gg}$ año-1) de emisiones totales $\mathrm{CO}_{2 \text {-Eq }}$ por lo que se reafirma que las Centrales Hidroeléctricas analizadas emiten GEI pero en escala reducida en comparación con las de Brasil y con el estándar de la ONU.

- Se recomienda la elaboración y ejecución de un programa que permita conocer el estado real de las emisiones de cualquier embalse, estableciendo medidas para reducir sus aportes de GEI.

- Las emisiones de gases de efecto invernadero en las centrales hidroeléctricas peruanas son relativamente bajas debido a que se encuentran en lugares donde el entorno es predominantemente seco y frio haciendo que la producción de limo y algas no generen $\mathrm{CO}_{2}$ que es el mayor contaminante.
[1] D. Veran y I, Vazquez. (2017, Diciembre 12). Avanzando y midiendo consumo y producción sostenible para una economía bajo en carbono en economías de ingresos medios y nuevos países industrializados en Peru. [En línea]. Disponible en: http://perulca.com/wpcontent/uploads/2019/04/documento b.pdf

[2] P. Parekh . (2011, Diciembre 2). Avances en el campo de las emisiones de embalses: Un Informe sobre la Investigación y las Directrices Recientes. [En línea]. Disponible en: https://www.internationalrivers.org/sites/default/files/attached

files/final_reservoiremissionsbriefing2011_esp_o_o.pdf

[3] M. Orizaola. "Una visión Global del Efecto Invernadero". Grado de maestro en educación primaria, Universidad de Cantabria, Cantabria, España, 2017. Disponible

https://archive.internationalrivers.org/node/2628

[4] W. Tep. (2016, Octubre 23). La Huella de Carbono y su Relación con el Efecto Invernadero. [En línea]. Disponible en: https://kanankab.wordpress.com/2016/10/23/la-huella-de-carbono-ysu-relacion-con-el-efecto-invernadero/

[5] J, Redondo. (2018,Enero 24). Centrales Hidroeléctricas - Qué Son, Tipos y Funcionamiento de las Centrales en España. [En línea]. Disponible en: https://erenovable.com/centrales-hidroelectricas/

[6] W. Sandoval. Presas y embalses. Sangolqui, Ecuador: Universidad de las Fuerzas Armadas-ESPE, 2018

[7] A. Palau. Análisis del ciclo de carbono en embalses y su posible efecto en el cambio climático: Aplicación al embalse de Susqueda (Río Ter, NE España). Valencia, España: Universidad Politécnica de Valencia, 2010

[8] Electroperu S.A. Memoria anual año 2017. Lima, Perú, marzo,2018

[9] GrañayMontero. (2020, Enero 17). Presa y túnel para la central hidroeléctrica Cerro del Águila. [En linea]. Disponible en: http://www.gym.com.pe/nuestros-proyectos/proyecto/proyectocentral-hidroelectrica-cerro-del-aguila

[10] GESTION (2020, Enero 17). [En línea]. Disponible en: https://gestion.pe/economia/empresas/central-hidroelectrica-chagllaus-1-200-millones-inicio-llenado-embalse-agua-99918-noticia/

[11] Enel. (2020, Enero 17). [En línea]. Disponible en: https://www.enel.pe/es/quienes-somos/a201611-huinco.html

[12] Celepsa. (2020, Enero 17). [En línea]. Disponible en: http://www.celepsa.com/las-hidroelectricas/

[13] Statkraft. (2020, Enero 18). [En línea]. Disponible en: https://www.statkraft.com.pe/acerca-de-statkraft/statkraft-en-

peru/central-hidroelectrica-cheves/

[14] ODEBRECHT. (2020, Enero 18). [En línea]. Disponible en: http://www.odebrecht.com.pe/negocios/infraestructura/obrasrealizadas/energia/central-hidroelectrica-charcani-v

[15] Endesa. (2020, Enero 20). [En linea]. Disponible en: http://www.endesa.com/

[16] EnerSur. Central Hidroeléctrica Yuncán. Huancavelica, Peru,2015. [17] ITAIPU BINACIONAL. (2017, Diciembre 29). La Itaipu: Eficiencia Que Redunda en Energía. [En línea]. Disponible en: https://www.itaipu.gov.py/es/sala-de-prensa/noticia/la-itaipueficiencia-que-redunda-en-energia

[18] IPCC. Directrices del IPCC de 2006 para los inventarios de gases de efecto invernadero. Volumen 4. Apéndice 2: emisiones de $\mathrm{CO}_{2}$ provenientes de las tierras inundadas: Base para su futuro metodológico. Francia,2006

[19] IPCC. Directrices del IPCC de 2006 para los inventarios de gases de efecto invernadero. Volumen 4. Apéndice 3: Emisiones de $\mathrm{CH}_{4}$ provenientes de las tierras inundadas: Base para su futuro metodológico Francia, 2006

[20] M.Demarty y J.Bastien. GHG emissions from hydroelectric reservoirs in tropical and equatorial regions: Review of 20 years of $\mathrm{CH}_{4}$ emission measurements Energy Policy, Montreal, Canada,2011

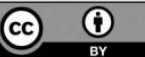

Los artículos publicados por TECNIA pueden ser compartidos a través de la licencia Creative Commons: CC BY 4.0. Permisos lejos de este alcance pueden ser consultados a través de correorevistas@uni.edu.pe

\section{REFERENCIAS}

